FACTA UNIVERSITATIS (NIŠ)

Ser. Math. Inform. Vol. 34, No 5 (2019), 903-925

https://doi.org/10.22190/FUMI1905903K

\title{
ON GENERALIZED FIBONACCI DIFFERENCE SPACE DERIVED FROM THE ABSOLUTELY $p$ - SUMMABLE SEQUENCE SPACES
}

\author{
Gülsen Kılınç
}

(C) 2019 by University of Niš, Serbia | Creative Commons Licence: CC BY-NC-ND

\begin{abstract}
In this study, it is specified the sequence space $l(F(r, s), p)$, (where $p=\left(p_{k}\right)$ is any bounded sequence of positive real numbers) and researched some algebraic and topological features of this space. Further, $\alpha-, \beta-, \gamma-$ duals and its Schauder Basis are given. The classes of matrix transformations from the space $l(F(r, s), p)$ to the spaces $l_{\infty}, c$, and $c_{0}$ are qualified. Additionally, acquiring qualifications of some other matrix transformations from the space $l(F(r, s), p)$ to the Euler, Riesz, difference, etc., sequence spaces is the other result of the paper.
\end{abstract}

Keywords. Matrix transformations; sequence space; Schauder Basis.

\section{Introduction}

In the first instance, let's remember some basic concept definitions in summability theory. The symbol $w$ denotes the space of all real or complex valued sequences. A subspace of $w$ is entitled sequence space. Some of the most known sequence spaces are $l_{\infty}, c, c_{0}$ and $l_{p}(1 \leq p \leq \infty)$ known as classical sequence spaces. The spaces which represented by these symbols are all bounded, convergent, null sequences and absolutely $p$-summable sequences, respectively. The spaces $l_{\infty}, c, c_{0}$ are Banach spaces with the norm

$$
\|z\|_{\infty}=\sup _{r \in \mathrm{N}}\left|z_{r}\right|
$$

and the space $l_{p}(1 \leq p \leq \infty)$ is Banach space with the norm

$$
\|z\|_{p}=\left(\sum_{r}\left|z_{r}\right|^{p}\right)^{\frac{1}{p}} .
$$

Received July 26, 2018; accepted June 25, 2019

2010 Mathematics Subject Classification. 46A45; 46B45, 46A35 
The spaces $b s$ and $c s$ are the space of the sequences that constitute the bounded and convergent series, respectively. The space $c_{0} s$ is defined

$$
c_{0} s=\left\{y=\left(y_{r}\right) \in w: \sum_{r} y_{r}=0\right\},
$$

the sequence space $b v$ defined by

$$
\left\{z=\left(z_{r}\right) \in w: \sum_{r=1}^{\infty}\left|z_{r}-z_{r-1}\right|<\infty\right\} .
$$

The space $b v_{0}$ denotes $b v_{0}=b v \cap c_{0}$.

To straightforwardness in representation, the symbol $\sum_{r}$ will be used instead of the symbol $\sum_{r=0}^{\infty}$ throughout this study. Also, the representations $e$ and $e^{(n)}$ denote $(1,1, \ldots)$ and the sequence $n$-th unit vector, respectively; where $n \in \mathrm{N}$ and $\mathrm{N}=\{0,1,2, \ldots\}$.

A sequence space $\vartheta$ with a linear topology is entitled a $K$-space ensured that each of the maps $p_{i}: \vartheta \rightarrow \mathrm{C}$ defined by $p_{i}(z)=z_{i}$ is continuous for all $i \in \mathrm{N}$, where $\mathrm{C}$ demonstrates the complex field. A $K$-space $\vartheta$ is entitled an $F K$-space ensured $\vartheta$ is a complete linear metric space. An $F K$-space whose topology is normable is entitled a $B K$-space (see [1]) which comprises $\Phi$, the set of all finitely nonzero sequences. [12]

If algebraic operations are continuous in a linear $T$ space fitted with a $\rho$ metric, then $(T, \rho)$ is entitled linear metric space. (see [27]). That is, when $\left(t_{n}\right)$ and $\left(z_{n}\right)$ are any two sequences in $T$ and $\left(k_{n}\right)$ is a sequence of scalars, then provision of conditions $\lim _{n \rightarrow \infty} \rho\left(t_{n}, t\right)=0, \lim _{n \rightarrow \infty} \rho\left(z_{n}, z\right)=0$ and $\lim _{n \rightarrow \infty} \rho\left(k_{n}\right)=k$ means provision of results $\lim _{n \rightarrow \infty} \rho\left(t_{n}+z_{n}, t+z\right)=0$ and $\lim _{n \rightarrow \infty} \rho\left(k_{n} t_{n}, k t\right)=0$. If $T$ linear metric space is complete, it is named Frechet sequence space [28].

Let $\nu$ and $\eta$ be a sequence space and $A=\left(a_{i j}\right)$ an infinite matrix of real or complex numbers and where $i, j \in \mathrm{N}$. If for each sequence $z=\left(z_{j}\right)$ in $\nu$, the $A$-transform of $z$ is in $\eta$, then $A$ - is called a matrix transformation from $\nu$ into $\eta$ and we demonstrate it by writing $A: \nu \rightarrow \eta$, where for all $i \in \mathrm{N}$,

$$
(A z)_{i}=\sum_{j} a_{i j} z_{j}
$$

The class of all matrices specified $A: \nu \rightarrow \eta$ is denoted by the notation $(\nu: \eta)$. The infinite matrix $A$ belongs to the class $(\nu: \eta)$ iff for each $i \in \mathrm{N}$, and every $z \in \nu$, $\sum_{j} a_{i j} z_{j}$ series are convergent and $(A z)_{i} \in \eta$.

The matrix domain $\nu_{A}$ of an infinite matrix $A$ in a sequence space $\nu$ is specified by

$$
\nu_{A}=\left\{z=\left(z_{k}\right) \in w: A z \in \nu\right\} .
$$

Let $U$ is an infinite matrix. If the constituents on the principal diagonal of $U$ are non- zero and the constituents on the top of the principal diagonal are zero, $U$ 
is entitled the triangular matrix. To study with triangular matrix domains has a special significance because of the features that these matrices have. Here are some of them: It is trivial that $Z(T y)=(Z T) y$ holds for the triangle matrices $Z, T$, and a sequence $y$. Further, a triangular matrix $U$ has an inverse matrix $U^{-1}=V$ that is only one and triangular. Then, $y=U(V y)=V(U y)$ holds for all $y \in w$. If $Z$ is triangle and $\nu$ is a $B K$-space, then $\nu_{Z}$ is also a $B K$-space with the norm given by $\|y\|_{\nu_{Z}}=\|Z y\|_{\nu}$ for all $y \in \nu_{Z}$.

If a function $h: T \rightarrow \mathrm{R}$ fulfills the the undermentioned conditions, for all $y, z \in T$

i) $h(y)=0$ if $y=\theta$,

ii) $h(y)=h(-y)$,

iii) $h(y+z) \leq h(y)+h(z)$

iv) $\left|\beta_{n}-\beta\right| \rightarrow 0$ and $h\left(y_{n}-y\right) \rightarrow 0$ imply $h\left(\beta_{n} y_{n}-\beta y\right) \rightarrow 0$, for all $\beta$ 's in $\mathrm{R}$ and all $y$ 's in $T$, where $\theta$ is the zero vector in the linear space $T$.

Then, a linear topological space $T$ defined on the real field $\mathrm{R}$ is entitled a paranormed space.

Let $\left(p_{r}\right)$ be a bounded sequence of exactly positive real numbers with $\sup _{r} p_{r}=$ $H$ and $M=\max \{1, H\}$. Then, absolutely $p$-summable sequences space $l(p)$ was specified by Maddox [13] (see also [14] and [15]) such as

$$
l(p)=\left\{y=\left(y_{r}\right) \in w: \sum_{r}\left|y_{r}\right|^{p_{r}}<\infty\right\},
$$

where $0 \leq p_{r} \leq H<\infty$. It is the complete space paranormed by

$$
h(y)=\left(\sum_{r}\left|y_{r}\right|^{p_{r}}\right)^{1 / M} .
$$

Throughout this study, $\mathcal{F}$ symbolizes the collection of all finite subsets of $\mathrm{N}$ and any constituent with a negative index is considered to be zero.

Let us bring to mind the sequence $\left(f_{n}\right)$ of Fibonacci numbers given by the linear iteration correlates: The first two constituents are taken as 1 and other index constituent are found by summing up the last two constituent that preceded it. Fibonacci numbers have many quirky features and practices in sciences, arts, and architecture. For instance, the ratio sequences of Fibonacci numbers converges to the golden ratio which is significant in sciences and arts. Also, some fundamental features of Fibonacci numbers $\left(f_{n}\right)$ are given as below:

$$
\begin{gathered}
\lim _{n \rightarrow \infty} \frac{f_{n+1}}{f_{n}}=\frac{1+\sqrt{5}}{2}=\phi \quad \text { (Golden Ratio), } \\
\sum_{k=0}^{n} f_{k}=f_{n+2}-1, \quad \text { for each } n \in \mathrm{N},
\end{gathered}
$$




$$
\sum_{k} \frac{1}{f_{k}}<\infty
$$

$$
f_{n-1} \cdot f_{n+1}-f_{n}^{2}=(-1)^{n+1} \quad \text { for each } \quad n \geq 1 \quad \text { (Cassini Formula). }
$$

The practice forming a new sequence space through the medium of the matrix domain of a special triangle has latterly been utilized by several authors in many exploratory papers. One of them is Fibonacci matrix defined Kara [4]. Now, let us familiarize aforementioned Fibonacci matrix $F=\left(f_{n k}\right)$

$$
f_{n k}=\left\{\begin{array}{cll}
-\frac{f_{n+1}}{f_{n}} & , \quad(k=n-1), \\
\frac{f_{n}}{f_{n+1}} & , \quad(k=n), \\
0 & , \quad(0 \leq k<n-1 \quad \text { or } k>n) .
\end{array}\right.
$$

It can be demonstrated readily that the matrix $\mathrm{F}$ is conservative, but it is neither regular nor coercive.

Let us acquaint some sequence spaces defined by the domain of a matrix and will be named in this work:

$$
b v_{\infty}=\left\{y=\left(y_{r}\right) \in w:\left(y_{r}-y_{r-1}\right) \in l_{\infty}\right\},
$$

was introduced by Başar and Altay [1].

$$
e_{\infty}^{r}=\left\{y=\left(y_{k}\right) \in w: E^{r} y \in l_{\infty}\right\},
$$

was introduced by Altay, Başar and Mursaleen [6].

$$
e_{c}^{r}=\left\{y=\left(y_{k}\right) \in w: E^{r} y \in c\right\},
$$

$$
e_{0}^{r}=\left\{y=\left(y_{k}\right) \in w: E^{r} y \in c_{0}\right\},
$$

were introduced by Altay, Başar [8]

$$
X_{\infty}=\left\{y=\left(y_{r}\right) \in w: C_{1} y \in l_{\infty}\right\},
$$

was introduced by $\mathrm{Ng}$ and Lee [2]

$$
\begin{gathered}
\widetilde{c}=\left\{y=\left(y_{r}\right) \in w: C_{1} y \in c\right\}, \\
\widetilde{c_{0}}=\left\{y=\left(y_{r}\right) \in w: C_{1} y \in c_{0}\right\},
\end{gathered}
$$

were introduced by Şengönül and Başar [9]

$$
r_{\infty}^{t}=\left\{y=\left(y_{k}\right) \in w: R^{t} y \in l_{\infty}\right\},
$$


was introduced by Altay and Başar [7].

$$
\begin{aligned}
& r_{c}^{t}=\left\{y=\left(y_{k}\right) \in w: R^{t} y \in c\right\}, \\
& r_{0}^{t}=\left\{y=\left(y_{k}\right) \in w: R^{t} y \in c_{0}\right\},
\end{aligned}
$$

was introduced by Altay and Başar [10].

$$
l_{\infty}(\widehat{F})=\left\{y=\left(y_{r}\right) \in w: F y \in l_{\infty}\right\},
$$

was introduced by Kara [4].

$$
\begin{gathered}
c(\widehat{F})=\left\{y=\left(y_{r}\right) \in w: F y \in c\right\}, \\
c_{0}(\widehat{F})=\left\{y=\left(y_{r}\right) \in w: F y \in c\right\},
\end{gathered}
$$

were introduced by Başarır et all [11].

$$
\begin{gathered}
c(\Delta)=\left\{y=\left(y_{r}\right) \in w:\left(y_{r}-y_{r+1}\right) \in c\right\}, \\
c_{0}(\Delta)=\left\{y=\left(y_{r}\right) \in w:\left(y_{r}-y_{r+1}\right) \in c_{0}\right\},
\end{gathered}
$$

was introduced by Kızmaz [5] where $E^{r}, C_{1}, R^{t}, F$ denote Euler mean of order $r$, arithmetic, Riesz means and Fibonacci matrix, respectively. Now, let us give aforementioned matrix methods:

$$
\begin{aligned}
& e_{n k}^{r}=\left\{\begin{array}{cc}
\left(\begin{array}{c}
n \\
k
\end{array}\right)(1-r)^{n-k} r^{k}, & 0 \leq k \leq n \\
0, & k>n
\end{array}\right. \\
& \delta_{n k}=\left\{\begin{array}{cc}
(-1)^{n-k}, & n-1 \leq k \leq n, \\
0, & 0 \leq k<n-1 \quad \text { or } \quad k>n,
\end{array}\right.
\end{aligned}
$$

the matrix $R^{t}=\left(r_{n k}^{t}\right)$ is specified by

$$
r_{n k}^{t}=\left\{\begin{array}{cc}
\frac{t_{k}}{T_{n}}, & 0 \leq k \leq n, \\
0, & k>n,
\end{array}\right.
$$

$\mathrm{Ng}$ and Lee specified the Cesaro sequence spaces $X_{p}$ and $X_{\infty}$ of non-absolute type by the set of the sequences whose $C$-transforms are in $l_{p}$ and $l_{\infty}$, respectively.

The matrix $C=\left(c_{n k}\right)$ is specified as follow:

$$
c_{n k}=\left\{\begin{array}{cc}
\frac{1}{n+1}, & 0 \leq k \leq n, \\
0, & k>n,
\end{array}\right.
$$


Now, let us present the matrix method which will be used to specify the sequence space which is subject of this work. It is entitled the generalized Fibonacci band matrix $F(r, s)$ specified by Candan at first [3]. It is formed by using Fibonacci sequence and non-zero real numbers $r$ and $s$.

$$
f_{n k}(r, s)=\left\{\begin{array}{cc}
s \frac{f_{n+1}}{f_{n}}, & k=n-1 \\
r \frac{f_{n}}{f_{n+1}}, & k=n \\
0, & \text { in other cases }
\end{array}\right.
$$

It can be seen that, the matrix $F(r, s)$ is degraded to the matrix $F$, for $r=1$ and $s=-1$. Therefore, the data acquired from the matrix $F(r, s)$ is more general than the data acquired from the $\widehat{F}$ matrix. The inverse $F^{-1}(r, s)$ of the matrix $F(r, s)$ is calculated as

$$
f_{n k}^{-1}(r, s)=\left\{\begin{array}{cc}
\frac{1}{r}\left(-\frac{s}{r}\right)^{n-k} \frac{f_{n+1}^{2}}{f_{k} \cdot f_{k+1}}, & 0 \leq k \leq n \\
0, & k>n
\end{array}\right.
$$

The other significant principal paper are here: [3], [16], [6], [17], [18], [26], [19], [20], [21], [22], [23], [24], [25], [31], [32], [34] .

This paper is organized as follow: In first chapter, it is familiarized generalized Fibonacci difference space $l(F(r, s), p)$ and is given its some algebraic and topological features. In second chapter, its $\alpha-, \beta-, \gamma-$ duals and Schauder Basis are determined. In third chapter, the qualifications of some other matrix transformations from the space $l(F(r, s), p)$ to the Euler, Riesz, difference, etc., sequence spaces are acquired.

\section{Some properties of the sequence space $l(F(r, s), p)$}

In this chapter, firstly, we familiarize the Fibonacci difference sequence spaces $l(F(r, s), p), l_{p}(F(r, s))$ as the set of all sequences whose $F(r, s)$-transforms are in the spaces $\ell(p)$ and $l_{p}$, respectively. Later, some algebraic and topological particularity of aforenamed these new spaces will be proved and a Schauder basis constructed. Let $p=\left(p_{k}\right)$ be any bounded sequence of positive real numbers. Then,

$$
l(F(r, s), p)=\left\{y=\left(y_{n}\right) \in w: \sum_{n}\left|r \frac{f_{n}}{f_{n+1}} y_{n}+s \frac{f_{n+1}}{f_{n}} y_{n-1}\right|^{p_{k}}<\infty\right\},
$$

where $0<p_{k} \leq H<\infty$. In the case, $p_{k}=p$ for all $k \in \mathrm{N}$, the space $l(F(r, s), p)$ is degraded to space $l_{p}(F(r, s))$, i.e., for $(p \geq 1)$

$$
l_{p}(F(r, s))=\left\{y=\left(y_{n}\right) \in w: \sum_{n}\left|r \frac{f_{n}}{f_{n+1}} y_{n}+s \frac{f_{n+1}}{f_{n}} y_{n-1}\right|^{p}<\infty\right\},
$$


By means of the notation of $(1.6)$, the spaces $l(F(r, s), p)$ and $l_{p}(F(r, s))$ can be redescribed as follows:

$$
l(F(r, s), p)=(l(p))_{F(r, s)} \text { and } l_{p}(F(r, s))=\left(l_{p}\right)_{F(r, s)} .
$$

Define the sequence $y=\left(y_{k}\right)$ by the $F(r, s)$-transform of a sequence $x=\left(x_{k}\right)$, i.e.,

for $n=0, y_{0}=r x_{0}$ and for $n \geq 1$

$$
y_{n}=(F(r, s) x)_{n}=r \frac{f_{n}}{f_{n+1}} x_{n}+s \frac{f_{n+1}}{f_{n}} x_{n-1},
$$

for all $k \in \mathrm{N}$. So, It can be acquired by a simplistic calculation that

$$
x_{n}=\sum_{j=0}^{n} \frac{1}{r}\left(-\frac{s}{r}\right)^{n-j} \frac{f_{n+1}^{2}}{f_{j} \cdot f_{j+1}} y_{j}
$$

Throughout the text, we assume that the sequences $x=\left(x_{k}\right)$ and $y=\left(y_{k}\right)$ are attached to the connection (2.4)

The following inequality will be employed throughout the paper. Let $p=\left(p_{k}\right)$ be a sequence of positive real numbers with $0<p_{k} \leq \sup _{k} p_{k}=H$, and let $D=$ $\max \left\{1,2^{H-1}\right\}$. Then, for the factorable sequences $\left(c_{k}\right)$ and $\left(d_{k}\right)$ in the complex plane, we have

$$
\left|c_{k}+d_{k}\right|^{p_{k}} \leq D\left(\left|c_{k}\right|^{p_{k}}+\left|d_{k}\right|^{p_{k}}\right)
$$

The other inequality is Minkowsky inequality, which will be employed in this paper. Its expression is here:

$$
\left(\sum_{k=1}^{\infty}\left|c_{k}+d_{k}\right|^{p}\right)^{1 / p} \leq\left(\sum_{k=1}^{\infty}\left|c_{k}\right|^{p}\right)^{1 / p}+\left(\sum_{k=1}^{\infty}\left|d_{k}\right|^{p}\right)^{1 / p}
$$

Theorem 2.1. $l(F(r, s), p)$ is a linear, complete and paranormed space with the $h$ function specified as

$$
h(x)=\left(\sum_{n}\left|r \frac{f_{n}}{f_{n+1}} x_{n}+s \frac{f_{n+1}}{f_{n}} x_{n-1}\right|^{p_{n}}\right)^{\frac{1}{M}},
$$

where $0<p_{n} \leq H<\infty$, for all $n \in N$.

Proof. Let $x, y \in l(F(r, s), p)$. Then

$$
\sum_{n}\left|r \frac{f_{n}}{f_{n+1}} x_{n}+s \frac{f_{n+1}}{f_{n}} x_{n-1}\right|^{p_{n}}<\infty,
$$




$$
\sum_{n}\left|r \frac{f_{n}}{f_{n+1}} y_{n}+s \frac{f_{n+1}}{f_{n}} y_{n-1}\right|^{p_{n}}<\infty .
$$

For $\lambda, \mu \in \mathrm{C}$, there comes into being integers $M_{\lambda}$, and $N_{\mu}$ such that $|\lambda| \leq M_{\lambda}$ and $|\mu| \leq N_{\mu}$. Employing Inequality (2.1), we have

$$
\begin{aligned}
& \sum_{n}\left|\lambda\left(r \frac{f_{n}}{f_{n+1}} x_{n}+s \frac{f_{n+1}}{f_{n}} x_{n-1}\right)+\mu\left(r \frac{f_{n}}{f_{n+1}} y_{n}+s \frac{f_{n+1}}{f_{n}} y_{n-1}\right)\right|^{p_{n}} \\
\leq & \sum_{n}\left(|\lambda|\left|r \frac{f_{n}}{f_{n+1}} x_{n}+s \frac{f_{n+1}}{f_{n}} x_{n-1}\right|\right)^{p_{n}} \\
& +\sum_{n}\left(|\mu|\left|r \frac{f_{n}}{f_{n+1}} y_{n}+s \frac{f_{n+1}}{f_{n}} y_{n-1}\right|\right)^{p_{n}} \\
\leq & D \cdot M_{\lambda}^{H} \sum_{n}\left(\left|r \frac{f_{n}}{f_{n+1}} x_{n}+s \frac{f_{n+1}}{f_{n}} x_{n-1}\right|\right)^{p_{n}} \\
& +D \cdot N_{\mu}^{H} \sum_{n}\left(\left|r \frac{f_{n}}{f_{n+1}} x_{n}+s \frac{f_{n+1}}{f_{n}} x_{n-1}\right|\right)^{p_{n}} \\
< & \infty .
\end{aligned}
$$

So that $\lambda x+\mu y \in l(F(r, s), p)$. This substantiates that $l(F(r, s), p)$ is a linear space.

Clearly, $h(x)=h(-x)$, for all $x \in l(F(r, s), p)$. It is unconcealed that $r \frac{f_{n}}{f_{n+1}} x_{n}+$ $s \frac{f_{n+1}}{f_{n}} x_{n-1}=0$, for $x=0$. Since $\frac{p_{n}}{M} \leq 1$, employing Minkowski Inequality, we have

$$
\begin{aligned}
h(x+y) & =\left[\sum_{n}\left|r \frac{f_{n}}{f_{n+1}}\left(x_{n}+y_{n}\right)+s \frac{f_{n+1}}{f_{n}}\left(x_{n-1}+y_{n-1}\right)\right|^{p_{n}}\right]^{\frac{1}{M}} \\
& =\left[\sum_{n}\left(\left|\left(r \frac{f_{n}}{f_{n+1}} x_{n}+s \frac{f_{n+1}}{f_{n}} x_{n-1}\right)+\left(r \frac{f_{n}}{f_{n+1}} y_{n}+s \frac{f_{n+1}}{f_{n}} y_{n-1}\right)\right|^{\frac{p_{n}}{M}}\right)^{M}\right]^{\frac{1}{M}} \\
& \leq\left[\sum_{n}\left(\left|r \frac{f_{n}}{f_{n+1}} x_{n}+s \frac{f_{n+1}}{f_{n}} x_{n-1}\right|^{\frac{p_{n}}{M}}+\left|r \frac{f_{n}}{f_{n+1}} y_{n}+s \frac{f_{n+1}}{f_{n}} y_{n-1}\right|^{\frac{p_{n}}{M}}\right)^{M}\right]^{\frac{1}{M}} \\
& \leq\left(\sum_{n}\left|r \frac{f_{n}}{f_{n+1}} x_{n}+s \frac{f_{n+1}}{f_{n}} x_{n-1}\right|^{p_{n}}\right)^{\frac{1}{M}}+\left(\sum_{n}\left|r \frac{f_{n}}{f_{n+1}} y_{n}+s \frac{f_{n+1}}{f_{n}} y_{n-1}\right|^{p_{n}}\right)^{\frac{1}{M}} \\
& =h(x)+h(y) .
\end{aligned}
$$


Also, since the inequality $|\alpha|^{p_{n}} \leq \max \left\{1,|\alpha|^{M}\right\}$ is ensured for $\alpha \in \mathrm{R}$, we get

$$
\begin{aligned}
h(\alpha x) & =\left[\sum_{n}\left|r \frac{f_{n}}{f_{n+1}}\left(\alpha x_{n}\right)+s \frac{f_{n+1}}{f_{n}}\left(\alpha x_{n-1}\right)\right|^{p_{n}}\right]^{\frac{1}{M}}, \\
& =\left(\sum_{n}|\alpha|^{p_{k}} \cdot\left|r \frac{f_{n}}{f_{n+1}} x_{n}+s \frac{f_{n+1}}{f_{n}} x_{n-1}\right|^{p_{n}}\right)^{\frac{1}{M}}, \\
& \leq \max \{1,|\alpha|\} \cdot h(x) .
\end{aligned}
$$

Let $\left(\alpha_{n}\right)$ be a sequence of scalars with $\alpha_{n} \rightarrow \alpha$, as $n \rightarrow \infty$ and $\left\{x^{(n)}\right\}_{n=0}^{\infty}$ be a sequence of components $x^{(n)} \in l(F(r, s), p)$ with $h\left(x^{(n)}-x\right) \rightarrow 0$, as $n \rightarrow \infty$. Then, we follow up that

$$
\begin{aligned}
0 \leq & h\left(\alpha_{n} x^{(n)}-\alpha x\right)=h\left(\alpha_{n} x^{(n)}-\alpha x^{(n)}+\alpha x^{(n)}-\alpha x\right) \\
& =h\left(\left(\alpha_{n}-\alpha\right) x^{(n)}+\alpha\left(x^{(n)}-x\right)\right) \\
& \leq h\left(\left(\alpha_{n}-\alpha\right) x^{(n)}\right)+h\left(\alpha\left(x^{(n)}-x\right)\right) \\
& =\left|\alpha_{n}-\alpha\right| \cdot h\left(x^{(n)}\right)+\max \{1,|\alpha|\} \cdot h\left(x^{(n)}-x\right) .
\end{aligned}
$$

If we combine the facts $\alpha_{n}-\alpha \rightarrow 0$, as $n \rightarrow \infty$ and $h\left(x^{(n)}-x\right) \rightarrow 0$,as $n \rightarrow$ $\infty$ with (2.11) we acquire that $h\left(\alpha_{n} x^{(n)}-\alpha x\right) \rightarrow 0$, as $n \rightarrow \infty$. That is, scalar multiplication is continuous. This shows that $h$ is a paranorm on $l(F(r, s), p)$. Moreover, if we assume $h(x)=0$, then we get

$$
\left|r \frac{f_{n}}{f_{n+1}} x_{n}+s \frac{f_{n+1}}{f_{n}} x_{n-1}\right|=0,
$$

for each $n \in \mathrm{N}$, we acquire that $x=\theta=(0,0,0, \ldots)$. It demonstrates that $h$ is total paranorm. Now, we indicate that $l(F(r, s), p)$ is complete. Let $\left(x^{(n)}\right)$ be any Cauchy sequence in $l(F(r, s), p)$, where $x^{(n)}=\left\{x_{1}^{(n)}, x_{2}^{(n)}, x_{3}^{(n)}, \ldots\right\}$. Then, for a given $\varepsilon>0$, there comes into being a positive integer $n_{0}(\varepsilon)$ such that $\left[h\left(x^{n}-x^{m}\right)\right]^{M}<\varepsilon^{M}$ for all $n, m>n_{0}(\varepsilon)$. Since for each immutable $k \in \mathrm{N}$

$$
\begin{aligned}
& \left|\left(F(r, s) x^{n}\right)_{k}-\left(F(r, s) x^{m}\right)_{k}\right|^{p_{k}} \leq \sum_{k}\left|\left(F(r, s) x^{n}\right)_{k}-\left(F(r, s) x^{m}\right)_{k}\right|^{p_{k}}, \\
= & \sum_{k}\left|\left(r \frac{f_{k}}{f_{k+1}} x_{k}^{(n)}+s \frac{f_{k+1}}{f_{k}} x_{k-1}^{(n)}\right)-\left(r \frac{f_{k}}{f_{k+1}} x_{k}^{(m)}+s \frac{f_{k+1}}{f_{k}} x_{k-1}^{(m)}\right)\right|^{p_{k}}, \\
= & \sum_{k}\left|\left(r \frac{f_{k}}{f_{k+1}}\left(x_{k}^{(n)}-x_{k}^{(m)}\right)+s \frac{f_{k+1}}{f_{k}}\left(x_{k-1}^{(n)}-x_{k-1}^{(m)}\right)\right)\right|^{p_{k}}, \\
= & {\left[h\left(x^{n}-x^{m}\right)\right]^{M}<\varepsilon^{M} . }
\end{aligned}
$$


For every $n, m>n_{0}(\varepsilon),\left\{\left(F(r, s) x^{0}\right)_{k},\left(F(r, s) x^{1}\right)_{k},\left(F(r, s) x^{2}\right)_{k}, \ldots\right\}$ is a Cauchy sequence of real numbers for every immutable $k \in \mathrm{N}$. Since $\mathrm{R}$ is complete, it is convergent,therefore we say

$$
\left(F(r, s) x^{n}\right)_{k} \rightarrow(F(r, s) x)_{k}
$$

as $n \rightarrow \infty$. Employing these infinitely many limits $(F(r, s) x)_{0},(F(r, s) x)_{1},(F(r, s) x)_{2}, \ldots$ we specify the sequence $\left\{(F(r, s) x)_{0},(F(r, s) x)_{1},(F(r, s) x)_{2}, \ldots\right\}$. For each $k \in \mathrm{N}$ and $n>n_{0}(\varepsilon)$

$$
\begin{aligned}
{\left[h\left(x^{n}-x\right)\right]^{M} } & =\sum_{k}\left|r \frac{f_{k}}{f_{k+1}}\left(x_{k}^{(n)}-x_{k}\right)+s \frac{f_{k+1}}{f_{k}}\left(x_{k-1}^{(n)}-x_{k-1}\right)\right|^{p_{k}} \\
& =\sum_{k}\left|\left(r \frac{f_{k}}{f_{k+1}} x_{k}^{(n)}+s \frac{f_{k+1}}{f_{k}} x_{k-1}^{(n)}\right)-\left(r \frac{f_{k}}{f_{k+1}} x_{k}+s \frac{f_{k+1}}{f_{k}} x_{k-1}\right)\right|^{p_{k}} \\
& =\sum_{k}\left|\left(F(r, s) x^{n}\right)_{k}-(F(r, s) x)_{k}\right|^{p_{k}}<\varepsilon^{M} .
\end{aligned}
$$

This shows that $\left(x^{n}-x\right) \in l(F(r, s), p)$. Since $l(F(r, s), p)$ is a linear space, we conclude that $x \in l(F(r, s), p)$. It follows that $x^{n} \rightarrow x$, as $n \rightarrow \infty$, in $l(F(r, s), p)$, it means that $l(F(r, s), p)$ is complete. Now, someone can readily check that the absolute feature does not verify on the space $l(F(r, s), p)$, that is

$$
\begin{aligned}
h(x) & =\left(\sum_{k}\left|r \frac{f_{k}}{f_{k+1}} x_{k}+s \frac{f_{k+1}}{f_{k}} x_{k-1}\right|^{p_{k}}\right)^{\frac{1}{M}} \neq\left(\sum_{k}\left|r \frac{f_{k}}{f_{k+1}}\right| x_{k}\left|+s \frac{f_{k+1}}{f_{k}}\right| x_{k-1}||^{p_{k}}\right)^{\frac{1}{M}} \\
& =h(|x|),
\end{aligned}
$$

where $|x|=\left(\left|x_{k}\right|\right)$. This says that $l(F(r, s), p)$ is the sequence space of non-absolute type.

Theorem 2.2. Convergence in $l(F(r, s), p)$ is strictly stronger than coordinatewise convergence, but the contrary isn't actual, ordinarily.

Proof. First, we indicate that $h\left(x^{n}-x\right) \rightarrow 0$, as $n \rightarrow \infty$ purportes $x_{k}^{(n)} \rightarrow x_{k}$, as $n \rightarrow \infty$, for all $k \in \mathrm{N}$. If we fix $k$, then we have

$$
\begin{aligned}
0 & \leq\left|\left(r \frac{f_{k}}{f_{k+1}} x_{k}^{(n)}+s \frac{f_{k+1}}{f_{k}} x_{k-1}^{(n)}\right)-\left(r \frac{f_{k}}{f_{k+1}} x_{k}+s \frac{f_{k+1}}{f_{k}} x_{k-1}\right)\right|^{p_{k}}, \\
& \leq \sum_{k}\left|\left(r \frac{f_{k}}{f_{k+1}} x_{k}^{(n)}+s \frac{f_{k+1}}{f_{k}} x_{k-1}^{(n)}\right)-\left(r \frac{f_{k}}{f_{k+1}} x_{k}+s \frac{f_{k+1}}{f_{k}} x_{k-1}\right)\right|^{p_{k}}, \\
& =\sum_{k}\left|r \frac{f_{k}}{f_{k+1}}\left(x_{k}^{(n)}-x_{k}\right)+s \frac{f_{k+1}}{f_{k}}\left(x_{k-1}^{(n)}-x_{k-1}\right)\right|^{p_{k}}, \\
& =\left[h\left(x^{n}-x\right)\right]^{M} .
\end{aligned}
$$


Hence, we have for $k=0$,

$$
\lim _{n \rightarrow \infty}\left|\left(r \frac{f_{0}}{f_{1}} x_{0}^{(n)}+s \frac{f_{1}}{f_{0}} x_{-1}^{(n)}\right)-\left(r \frac{f_{0}}{f_{1}} x_{0}+s \frac{f_{1}}{f_{0}} x_{-1}\right)\right|=0,
$$

that is, $\left|r \frac{f_{0}}{f_{1}}\left[x_{0}^{(n)}-x_{0}\right]\right| \rightarrow 0$, as $n \rightarrow \infty$ and $\frac{f_{0}}{f_{1}}=1$ and $r \frac{f_{0}}{f_{1}}=r \neq 0$, then $\left|x_{0}^{(n)}-x_{0}\right| \rightarrow 0$ as $n \rightarrow \infty$. Likewise, for each $k \in \mathrm{N}$, we have $\left|x_{k}^{(n)}-x_{k}\right| \rightarrow 0$, as $n \rightarrow \infty$. Now, we demonstrate that the contrary isn't actual, ordinarily. We theorize $x_{k}^{(n)} \rightarrow x_{k}$, as $n \rightarrow \infty$. Then, there comes into being an $N \in \mathrm{N}$, such that $\left|x_{k}^{(n)}-x_{k}\right|<1$, for each immutable $k$ and for all $n \geq N$. Therefore, we see that,

$$
\begin{aligned}
& \leq \leq h\left(x^{n}-x\right)=\left(\sum_{k}\left|r \frac{f_{k}}{f_{k+1}}\left(x_{k}^{(n)}-x_{k}\right)+s \frac{f_{k+1}}{f_{k}}\left(x_{k-1}^{(n)}-x_{k-1}\right)\right|^{p_{k}}\right)^{\frac{1}{M}}, \\
= & \left\{\sum_{k}\left[\left|r \frac{f_{k}}{f_{k+1}}\left(x_{k}^{(n)}-x_{k}\right)+s \frac{f_{k+1}}{f_{k}}\left(x_{k-1}^{(n)}-x_{k-1}\right)\right|^{\frac{p_{k}}{M}}\right]^{M},\right. \\
\leq & \left\{\sum_{k}\left[\left|r \frac{f_{k}}{f_{k+1}}\left(x_{k}^{(n)}-x_{k}\right)\right|^{\frac{p_{k}}{M}}+\left|s \frac{f_{k+1}}{f_{k}}\left(x_{k-1}^{(n)}-x_{k-1}\right)\right|^{\frac{p_{k}}{M}}\right]^{M},\right. \\
\leq & {\left[\sum_{k}\left|r \frac{f_{k}}{f_{k+1}}\left(x_{k}^{(n)}-x_{k}\right)\right|^{p_{k}}\right]^{\frac{1}{M}}+\left[\sum_{k}\left|s \frac{f_{k+1}}{f_{k}}\left(x_{k-1}^{(n)}-x_{k-1}\right)\right|^{p_{k}}\right]^{\frac{1}{M}}, } \\
\leq & \left(\sum_{k}\left|r \frac{f_{k}}{f_{k+1}}\right|^{p_{k}} \cdot\left|x_{k}^{(n)}-x_{k}\right|^{p_{k}}\right)^{\frac{1}{M}}+\left(\sum_{k}\left|s \frac{f_{k+1}}{f_{k}}\right|^{p_{k}} \cdot\left|x_{k-1}^{(n)}-x_{k-1}\right|^{p_{k}}\right)^{\frac{1}{M}}, \\
\leq & \left(\sum_{k}\left|r \frac{f_{k}}{f_{k+1}}\right|^{p_{k}}\right)^{\frac{1}{M}}+\left(\sum_{k}\left|s \frac{f_{k+1}}{f_{k}}\right|^{p_{k}}\right)^{\frac{1}{M}}
\end{aligned}
$$

for all $k$ and $N$. Since $\left|\frac{f_{k+1}}{f_{k}}\right| \rightarrow 1,6 \Rightarrow\left|s \frac{f_{k+1}}{f_{k}}\right| \rightarrow|s| .1,6$ and $\left|\frac{f_{k}}{f_{k+1}}\right| \rightarrow 0,6 \Rightarrow$ $\left|r \frac{f_{k}}{f_{k+1}}\right| \rightarrow|r| .0,6$ as $k \rightarrow \infty$. In (2.15), $h\left(x^{n}-x\right)$ doesn't convergence for each immutable $k \in \mathrm{N}$ and for all $n \geq N$. This purports that the contrary isn't actual. Let us consider the elements of the sequence $x^{n}$ be equal, then we follow up that $h\left(x^{n}-x\right)=0$, that is to say that coordinatewise convergence requires convergence. Hence, we can say that the contrary is not actual, ordinarily.

Theorem 2.3. $l(F(r, s), p)$ is a $K$-space.

Proof. Firstly, we show that $q_{i}(x)=x_{i}$ is linear for all $i \in \mathrm{N}$. Let $x=\left(x_{i}\right), y=$ $\left(y_{i}\right) \in l(F(r, s), p)$ and $\alpha \in \mathrm{C}$. Then, we get

$$
q_{i}(x+y)=(x+y)_{i}=x_{i}+y_{i}=q_{i}(x)+q_{i}(y)
$$


and

$$
q_{i}(\alpha x)=(\alpha x)_{i}=\alpha x_{i}=\alpha q_{i}(x)
$$

for all $i \in \mathrm{N}$. Hence $q_{i}$ is linear. Now, we substantiate that $q_{i}$ is continuous. For this, it is sufficient to show that $q_{i}$ is bounded. Let $x=\left(x_{i}\right) \in l(F(r, s), p)$ be any vector. Then, since $\left|q_{i}(x)\right|=\left|x_{i}\right|$ for all $i \in \mathrm{N}$, one can see that

$$
\begin{aligned}
\left\|q_{i}\right\| & =\sup _{x \neq 0} \frac{\left|q_{i}(x)\right|}{\|x\|_{l(F(r, s), p)}}=\sup _{x \neq 0} \frac{\left|x_{i}\right|}{\|x\|_{l(F(r, s), p)}}, \\
& \leq \sup _{x \neq 0} \frac{\|x\|_{l(F(r, s), p)}}{\|x\|_{l(F(r, s), p)}}=1<\infty,
\end{aligned}
$$

i.e., $q_{i}$ is bounded. Hence, $q_{i}$ is a linear and continuous operator. That is to say that $l(F(r, s), p)$ is a $K$-space.

Theorem 2.4. $l(F(r, s), p)$ is an $F K$-space.

Proof. It is easy to see by Theorem (2.1) and Theorem (2.2) that $l(F(r, s), p)$ is complete sequence space and convergence requires coordinatewise convergence. Hence, $l(F(r, s), p)$ is an $F K$-space.

Theorem 2.5. $l_{p}(F(r, s))$ is linear space and a BK-space with the following norm

$$
\|x\|=\left(\sum_{n}\left|r \frac{f_{n}}{f_{n+1}} x_{n}+s \frac{f_{n+1}}{f_{n}} x_{n-1}\right|^{p}\right)^{1 / p},
$$

where $x=\left(x_{n}\right) \in l_{p}(F(r, s))$ and $1 \leq p<\infty$.

Proof. it will not be done because the proof of linearity of the space is routine operations. Because $l_{p}$ is a $B K$-space with known norm and $F(r, s)$ is a triangle matrix, according to Theorem 4.3.2 of Wilansky [28], we acquire that $l_{p}(F(r, s))$ is a $B K$-space.

Theorem 2.6. When $p$ fulfilled the condition $1 \leq p<\infty$. The newly specified sequence space $C$ is a $B K$-space with the norm $\|x\|_{l_{p}(F(r, s))}=\|f(r, s) x\|_{p}$, in other words,

$$
\|x\|_{l_{p}(F(r, s))}=\left(\sum_{n}\left|F_{n}(r, s) x\right|^{p}\right)^{\frac{1}{p}}, \quad 1 \leq p<\infty .
$$

Theorem 2.7. $l_{p}(F(r, s))$ is a Frechet space. 
Proof. To avoid the repetition of the similar expressions, we demonstrate that the algebraic operations are continuous in the space $l_{p}(F(r, s))$. Let $\left(x_{n}\right)$ and $\left(y_{n}\right)$ be two sequences in $l_{p}(F(r, s))$ and $\left(\alpha_{n}\right)$ be a sequence of scalars such that $d\left(x_{n}, x\right) \rightarrow$ $0, d\left(y_{n}, y\right) \rightarrow 0$ and $\alpha_{n} \rightarrow \alpha$, as $n \rightarrow \infty$. Then, we get that

$$
\begin{aligned}
& 0 \leq \lim _{n \rightarrow \infty} d\left(x_{n}+y_{n}, x+y\right), \\
= & \lim _{n \rightarrow \infty}\left(\left\|\left(x_{n}+y_{n}\right)-(x+y)\right\|\right) \\
\leq & \lim _{n \rightarrow \infty}\left(\left\|x_{n}-x\right\|+\left\|y_{n}-y\right\|\right) \\
= & \lim _{n \rightarrow \infty} d\left(x_{n}, x\right)+\lim _{n \rightarrow \infty} d\left(y_{n}, y\right) \\
= & 0 \\
& 0 \leq \lim _{n \rightarrow \infty} d\left(\alpha_{n} x_{n}, \alpha x\right) \\
= & \lim _{n \rightarrow \infty}\left\|\alpha_{n} x_{n}-\alpha x\right\| \\
= & \lim _{n \rightarrow \infty}\left\|\left(\alpha_{n}-\alpha\right) x_{n}+\alpha\left(x_{n}-x\right)\right\| \\
\leq & \lim _{n \rightarrow \infty}\left(\left|\alpha_{n}-\alpha\right|\left\|x_{n}\right\|+|\alpha|\left\|x_{n}-x\right\|\right) \\
= & \lim _{n \rightarrow \infty}\left|\alpha_{n}-\alpha\right|\left\|x_{n}\right\|+|\alpha| \lim _{n \rightarrow \infty} d\left(x_{n}, x\right) \\
= & 0
\end{aligned}
$$

It is ready to see from (2.20) and (2.21) that the algebraic operations are continuous on the linear metric space $l_{p}(F(r, s))$. Hence $l_{p}(F(r, s))$ is a Frechet space.

With the notation of (2.4), the transformation $T$ specified from $l(F(r, s), p)$ to $l(p)$ by $x \rightarrow y=T x$ is linear bijection, so we have the following:

Corollary 2.1. The sequence space $l(F(r, s), p)$ of the non-absolute type is linearly paranorm isomorphic to the space $l(p)$, where $0<p_{k} \leq H<\infty$, for all $k \in N$.

Due to the well known fact that the matrix domain $\nu_{A}$ of the normed sequence space denoted by $\nu$, has got a base iff $\nu$ has got a base, whenever a matrix $A$ is a triangle [31] (remark 2.4). Accordingly, we can give the following result:

Corollary 2.2. Let $0<p_{k} \leq H<\infty$ and $\lambda_{k}=(F x)_{k}$, for all $k \in N$. Specify the sequence $b^{(k)}=\left\{b_{n}^{(k)}\right\}_{n \in N}$ of the elements of the spaces $l(F(r, s), p)$ by

$$
b_{n}^{(k)}=\left\{\begin{array}{cc}
\frac{1}{r}\left(-\frac{s}{r}\right)^{k-n} \frac{f_{k+1}^{2}}{f_{n} \cdot f_{n+1}}, & 0 \leq n \leq k \\
0, & n>k
\end{array}\right.
$$

for every immutable $k \in N$. Then, the sequence $\left\{b^{(k)}\right\}_{k \in N}$ is a basis for the space $l(F(r, s), p)$ and any $x \in l(F(r, s), p)$ has a sole representation of the shape

$$
x=\sum_{k} \lambda_{k} b^{(k)}
$$




\section{The $\alpha-, \beta-, \gamma-$ Duals of the space $l(F(r, s), p)$}

The $\alpha-, \beta$-and $\gamma$ duals of the sequence space $X$ are specified as follows:

If $x$ and $y$ are sequences and $X$ and $Y$ are subsets of $w$, then, we write $x . y=$ $\left(x_{k} y_{k}\right)_{k=0}^{\infty}$, and

$$
\begin{gathered}
x^{-1} * Y=\{a \in w: a x \in Y\} \\
M(X, Y)=\bigcap_{x \in X} x^{-1} * Y=\{a \in w: a x \in Y, \text { for all } x \in X\},
\end{gathered}
$$

for the multiplier space of $X$ and $Y$. One can readily follow up for a sequence space $\mathrm{Z}$ with $Y \subset Z$ and $M(X, Y) \subset M(X, Z)$ that the containments $M(X, Y) \subset M(Z, Y)$ hold. The $\alpha-, \beta-$, and $\gamma-$ duals of a sequence space $X$, which are, respectively, denoted by

$$
X^{\alpha}=M\left(X, l_{1}\right), X^{\beta}=M(X, c s) \text { and } X^{\gamma}=M(X, b s)
$$

It is obvious that $X^{\alpha} \subset X^{\beta} \subset X^{\gamma}$. Also, it can be readily seen that the containments $X^{\alpha} \subset Y^{\alpha}, \quad X^{\beta} \subset Y^{\beta}$ and $X^{\gamma} \subset Y^{\gamma}$ hold, whenever $Y \subset X$.

Lemma 3.1. [30] Let $A=\left(a_{n k}\right)$ be an infinite matrix over the complex field. The following expressions are ensured:

i) Let $0<p_{k} \leq 1$ for all $k \in N$. Then, an infinite matrix $A$ transforms all sequences belong to $l(p)$ into $l_{1}$ iff

$$
\sup _{N \in \mathcal{F}} \sup _{k \in N}\left|\sum_{n \in N} a_{n k}\right|^{p_{k}}<\infty .
$$

ii) Let $1<p_{k} \leq H<\infty$, for all $k \in N$. Then, an infinite matrix $A$ transforms all sequences belong to $l(p)$ into $l_{1}$ iff there comes into being an integer $B>1$ such that

$$
\sup _{N \in \mathcal{F}} \sum_{k}\left|\sum_{n \in N} a_{n k} B^{-1}\right|^{q_{k}}<\infty .
$$

Lemma 3.2. [29] Let $A=\left(a_{n k}\right)$ be an infinite matrix over the complex field. The following expressions ensure:

i) Let $0<p_{k} \leq 1$ for all $k \in N$. Then, an infinite matrix $A$ transforms all sequences belong to $l(p)$ into $l_{\infty}$ iff

$$
\sup _{n, k \in N}\left|a_{n k}\right|^{p_{k}}<\infty
$$

ii) Let $1<p_{k} \leq H<\infty$, for all $k \in N$. Then, an infinite matrix $A$ transforms all sequences belong to $l(p)$ into $l_{\infty}$ iff there comes into being an integer $B>1$ such that

$$
\sup _{n \in N} \sum_{k}\left|a_{n k} B^{-1}\right|^{q_{k}}<\infty
$$


Lemma 3.3. [29] Let $A=\left(a_{n k}\right)$ be an infinite matrix over the complex field and $0<p_{k} \leq H<\infty$ for all $k \in N$. Then, an infinite matrix $A$ transforms all sequences belong to $l(p)$ into $c$ iff (3.6), (3.7) and for all $k \in N$

$$
\lim _{n \rightarrow \infty} a_{n k}=\beta_{k}
$$

ensures.

Let us specify the following sets:

$$
E_{1}=\left\{a=\left(a_{k}\right) \in w: \sup _{N \in \mathcal{F}} \sup _{k \in \mathrm{N}}\left|\sum_{n \in \mathrm{N}} \frac{1}{r}\left(-\frac{s}{r}\right)^{n-k} \frac{f_{n+1}^{2}}{f_{k} \cdot f_{k+1}} a_{n}\right|^{p_{k}}<\infty\right\}
$$

$$
E_{2}=\cup_{B>1}\left\{a=\left(a_{k}\right) \in w: \sup _{n \in \mathcal{F}} \sum_{k}\left|\sum_{n \in \mathrm{N}} \frac{1}{r}\left(-\frac{s}{r}\right)^{n-k} \frac{f_{n+1}^{2}}{f_{k} \cdot f_{k+1}} a_{n} \cdot B^{-1}\right|^{q_{k}}<\infty\right\}
$$

$$
E_{3}=\left\{a=\left(a_{k}\right) \in w: \sup _{k, n \in \mathrm{N}}\left|\sum_{j=k}^{n} \frac{1}{r}\left(-\frac{s}{r}\right)^{j-k} \frac{f_{j+1}^{2}}{f_{k} \cdot f_{k+1}} a_{j}\right|^{p_{k}}<\infty\right\}
$$

$$
E_{4}=\left\{a=\left(a_{k}\right) \in w: \sum_{j=k}^{\infty} \frac{1}{r}\left(-\frac{s}{r}\right)^{j-k} \frac{f_{j+1}^{2}}{f_{k} \cdot f_{k+1}} a_{j}<\infty\right\}
$$

$$
E_{5}=\cup_{B>1}\left\{a=\left(a_{k}\right) \in w: \sup _{n \in \mathrm{N}} \sum_{k}\left|\sum_{j=k}^{n} \frac{1}{r}\left(-\frac{s}{r}\right)^{j-k} \frac{f_{j+1}^{2}}{f_{k} \cdot f_{k+1}} a_{j} \cdot B^{-1}\right|^{q_{k}}<\infty\right\}
$$

Since proof of the first chapter of the following theorems can be made similar to the proof of the second chapter, we will only give proof of the second part.

Theorem 3.1. The following expressions are ensured:

i) Let $0<p_{k} \leq 1$ for all $k \in N$. Then,

$$
\{l(F(r, s), p)\}^{\alpha}=E_{1}
$$

ii) Let $1<p_{k} \leq H<\infty$, for all $k \in N$. Then,

$$
\{l(F(r, s), p)\}^{\alpha}=E_{2}
$$


Proof. Let us take any $a=\left(a_{n}\right) \in w$. By employing (2.5) we acquire that, for all $n \in \mathrm{N}$

$$
a_{n} x_{n}=\sum_{k=0}^{n}\left(\frac{1}{r}\right)\left(-\frac{s}{r}\right)^{n-k} \frac{f_{n+1}^{2}}{f_{k} \cdot f_{k+1}} a_{n} \cdot y_{k}=(E y)_{n},
$$

where $E=\left(e_{n k}\right)$ is specified by

$$
e_{n k}=\left\{\begin{array}{cc}
\left(\frac{1}{r}\right)\left(-\frac{s}{r}\right)^{n-k} \frac{f_{n+1}^{2}}{f_{k} \cdot f_{k+1}} a_{n}, & 0 \leq k \leq n, \\
0, & k>n,
\end{array}\right.
$$

for all $k, n \in \mathrm{N}$. Thus, we follow up by unification (3.16) with the proviso (3.5). of part (ii) of Lemma 3.1 that $a x=\left(a_{n} x_{n}\right) \in l_{1}$ whenever $x=\left(x_{k}\right) \in l(F(r, s), p)$ iff $E y \in l_{1}$, whenever $y=\left(y_{k}\right) \in l(F(r, s), p)$. This leads to fact that $\{l(F(r, s), p)\}^{\alpha}=$ $E_{2}$, as claimed.

Theorem 3.2. The following expressions are ensured:

i) Let $0<p_{k} \leq 1$ for all $k \in N$. Then,

$$
\{l(F(r, s), p)\}^{\beta}=E_{3} \cap E_{4} .
$$

ii) Let $1<p_{k} \leq H<\infty$, for all $k \in N$. Then,

$$
\{l(F(r, s), p)\}^{\beta}=E_{4} \cap E_{5} .
$$

Proof. Take any $a=\left(a_{j}\right) \in w$, then, one can acquire by (2.5) that

$$
\begin{gathered}
\sum_{j=0}^{n} a_{j} x_{j}=\sum_{j=0}^{n}\left(\sum_{k=0}^{j} \frac{1}{r}\left(-\frac{s}{r}\right)^{j-k} \frac{f_{j+1}^{2}}{f_{k} \cdot f_{k+1}} y_{k}\right) a_{j} \\
=\sum_{k=0}^{n}\left(\sum_{j=k}^{n} \frac{1}{r}\left(-\frac{s}{r}\right)^{j-k} \frac{f_{j+1}^{2}}{f_{k} \cdot f_{k+1}} a_{j}\right) y_{k} \\
=(D y)_{n},
\end{gathered}
$$

for all $n \in \mathrm{N}$, where $D=\left(d_{n k}\right)$ is specified by

$$
d_{n k}=\left\{\begin{array}{cc}
\sum_{j=k}^{n}\left(\frac{1}{r}\right)\left(-\frac{s}{r}\right)^{j-k} \frac{f_{j+1}^{2}}{f_{k} \cdot f_{k+1}} a_{j}, & 0 \leq k \leq n, \\
0, & k>n,
\end{array}\right.
$$

for all $n, k \in \mathrm{N}$. Thus, we make inferences from Lemma (3.3) with (3.20) that $a x=\left(a_{j} x_{j}\right) \in c s$ whenever $x=\left(x_{j}\right) \in l(F(r, s), p)$ iff $D y \in c$, whenever $y=$ $\left(y_{k}\right) \in l(p)$. Therefore, we derive from (3.7) and (3.8) that

$$
\sup _{n \in \mathrm{N}} \sum_{k}\left|\sum_{j=k}^{n} \frac{1}{r}\left(-\frac{s}{r}\right)^{j-k} \frac{f_{j+1}^{2}}{f_{k} \cdot f_{k+1}} a_{j} \cdot B^{-1}\right|^{q_{k}}<\infty,
$$




$$
\sum_{j=k}^{\infty} \frac{1}{r}\left(-\frac{s}{r}\right)^{j-k} \frac{f_{j+1}^{2}}{f_{k} \cdot f_{k+1}} a_{j}<\infty .
$$

This shows that $\{l(F(r, s), p)\}^{\beta}=E_{4} \cap E_{5}$

Theorem 3.3. The following expressions are ensured:

i) Let $0<p_{k} \leq 1$ for all $k \in N$. Then,

$$
\{l(F(r, s), p)\}^{\gamma}=E_{3} .
$$

ii) Let $1 \leq p_{k} \leq H<\infty$, for all $k \in N$. Then,

$$
\{l(F(r, s), p)\}^{\gamma}=E_{5} .
$$

Proof. From Lemma (3.2) and (3.20), we acquire that $a x=\left(a_{j} x_{j}\right) \in b s$ whenever $x=\left(x_{j}\right) \in l(F(r, s), p)$ iff $D y \in l_{\infty}$, whenever $y=\left(y_{k}\right) \in l(p)$, where $\mathrm{D}=\left(d_{n k}\right)$ is acquired by (3.21). Therefore, we acquire (3.6) and (3.7) that

$$
\{l(F(r, s), p)\}^{\gamma}= \begin{cases}E_{3}, & p_{k} \leq 1, \\ E_{5}, & p_{k}>1\end{cases}
$$

as desired.

\section{Some Matrix Transformations on the space $l(F(r, s), p)$}

In this section, we characterize some matrix transformations on the space $l(F(r, s), p)$, since the cases $0<p_{k} \leq 1$ and $1<p_{k} \leq H<\infty$ are integrated. The following theorem gives the exact provisos of the general case $0<p_{k} \leq H<\infty$. We consider only the case $1<p_{k} \leq H<\infty$ and omit the proof of the case $0<p_{k} \leq 1$, since it can be ensured in an alike way.

Theorem 4.1. The following expressions are ensured:

i) Let $0<p_{k} \leq 1$ for all $k \in N$. Then, an infinite matrix $A$ transforms all sequences belong to $l(F(r, s), p)$ into $l_{\infty}$ iff

$$
\begin{gathered}
\sup _{k, n \in N}\left|\sum_{j=k}^{\infty} \frac{1}{r}\left(-\frac{s}{r}\right)^{j-k} \frac{f_{j+1}^{2}}{f_{k} \cdot f_{k+1}} a_{n j}\right|^{p_{k}}<\infty, \\
\sum_{j=k}^{\infty} \frac{1}{r}\left(-\frac{s}{r}\right)^{j-k} \frac{f_{j+1}^{2}}{f_{k} \cdot f_{k+1}} a_{n j}<\infty .
\end{gathered}
$$

ii) Let $1<p_{k} \leq H<\infty$, for all $k \in N$. Then, an infinite matrix $A$ transforms all sequences belong to $l(F(r, s), p)$ into $l_{\infty}$ iff (4.1) ensures and there comes into being an integer $B>1$ such that

$$
\sup _{n \in N} \sum_{k}\left|\sum_{j=k}^{\infty} \frac{1}{r}\left(-\frac{s}{r}\right)^{j-k} \frac{f_{j+1}^{2}}{f_{k} \cdot f_{k+1}} a_{n j} B^{-1}\right|^{q_{k}}<\infty .
$$


Proof. Let $A \in\left(l(F(r, s), p): l_{\infty}\right)$ and $1<p_{k} \leq H<\infty$, for all $k \in \mathrm{N}$. Then, $A x$

comes into being for every $x \in l(F(r, s), p)$ and this implies that $A_{n} \in\{l(F(r, s), p)\}^{\beta}$ for each immutable $n \in \mathrm{N}$. Therefore, the necessities of (4.2) and (4.3) are immediate.

Conversely, suppose that the provisos (4.2) and (4.3) ensure, and take any $x \in$ $l(F(r, s), p)$. Since, $A_{n} \in\{l(F(r, s), p)\}^{\beta}$, for each $n \in \mathrm{N}, A x$ comes into being. By employing (2.5), we acquire that

$$
\sum_{j=0}^{m} a_{n j} x_{j}=\sum_{j=0}^{m} \sum_{k=0}^{j} \frac{1}{r}\left(-\frac{s}{r}\right)^{j-k} \frac{f_{j+1}^{2}}{f_{k} \cdot f_{k+1}} y_{k} a_{n j}=\sum_{k=0}^{m} \sum_{j=k}^{m} \frac{1}{r}\left(-\frac{s}{r}\right)^{j-k} \frac{f_{j+1}^{2}}{f_{k} \cdot f_{k+1}} a_{n j} y_{k},
$$

for all $m, n \in \mathrm{N}$. Taking into account the hypothesis, we reproduce from (4.4), as $m \rightarrow \infty$ that for all $n \in \mathrm{N}$,

$$
\sum_{j} a_{n j} x_{j}=\sum_{k} \sum_{j=k}^{\infty} \frac{1}{r}\left(-\frac{s}{r}\right)^{j-k} \frac{f_{j+1}^{2}}{f_{k} \cdot f_{k+1}} a_{n j} y_{k}
$$

By integrating (4.5) and the inequality which ensures for any complex numbers $a, b$ and any $B>0$,

$$
|a b| \leq B\left(\left|a B^{-1}\right|^{q}+|b|^{p}\right),
$$

where $p>1$ and $p^{-1}+q^{-1}=1$, we acquire that,

$$
\begin{aligned}
\sup _{n \in \mathrm{N}}\left|\sum_{j} a_{n j} x_{j}\right| & =\sup _{n \in \mathrm{N}}\left|\sum_{k} \sum_{j=k}^{\infty} \frac{1}{r}\left(-\frac{s}{r}\right)^{j-k} \frac{f_{j+1}^{2}}{f_{k \cdot} \cdot f_{k+1}} a_{n j} y_{k}\right| \\
& \leq \sup _{n \in \mathrm{N}} \sum_{k}\left|\sum_{j=k}^{\infty} \frac{1}{r}\left(-\frac{s}{r}\right)^{j-k} \frac{f_{j+1}^{2}}{f_{k \cdot f} \cdot f_{k+1}} a_{n j} y_{k}\right|, \\
& \leq \sup _{n \in \mathrm{N}} \sum_{k} B\left(\left|\sum_{j=k}^{\infty} \frac{1}{r}\left(-\frac{s}{r}\right)^{j-k} \frac{f_{j+1}^{2}}{f_{k} \cdot f_{k+1}} a_{n j} B^{-1}\right|^{q_{k}}+\left|y_{k}\right|^{p_{k}}\right), \\
& =B\left(\sup _{n \in \mathrm{N}} \sum_{k}\left|\sum_{j=k}^{\infty} \frac{1}{r}\left(-\frac{s}{r}\right)^{j-k} \frac{f_{j+1}^{2}}{f_{k} \cdot f_{k+1}} a_{n j} B^{-1}\right|^{q_{k}}+\sup _{n \in \mathrm{N}} \sum_{k}\left|y_{k}\right|^{p_{k}}\right) \\
& <\infty .
\end{aligned}
$$

This shows that $A x \in l_{\infty}$.

Theorem 4.2. The following expressions are ensured: 
i) Let $0<p_{k} \leq 1$ for all $k \in N$. Then, an infinite matrix $A$ transforms all sequences belong to $l(F(r, s), p)$ into $c$ iff (4.1) and (4.2) ensure and there is a sequence $\alpha=\left(\alpha_{k}\right)$ of scalars such that

$$
\lim _{n \rightarrow \infty} \sum_{j=k}^{\infty} \frac{1}{r}\left(-\frac{s}{r}\right)^{j-k} \frac{f_{j+1}^{2}}{f_{k} \cdot f_{k+1}} a_{n j}=\alpha_{k},
$$

for all $k \in N$.

ii) Let $1<p_{k} \leq H<\infty$, for all $k \in N$. Then, an infinite matrix $A$ transforms all sequences belong to $l(F(r, s), p)$ into $c$ iff (4.2), (4.3) and (4.7), ensure.

Proof. Let $A \in(l(F(r, s), p): c)$ and $1<p_{k} \leq H<\infty$ for all $k \in \mathrm{N}$. Then, since the containment $c \subset l_{\infty}$ ensures, the neccesities of (4.2) and (4.3) are immediately acquired from Theorem (4.). To ensure the the neccesities of (4.7), consider the sequence $b^{(k)}$ defined by $(2.22)$ which belongs to the space $l(F(r, s), p)$ for every immutable $k \in \mathrm{N}$. Since, the $A$ - transform of every $x \in l(F(r, s), p)$ comes into being and is in $c$ by the hypothesis, we have

$$
A b^{(k)}=\left(\sum_{j=0}^{\infty} a_{i j} b_{j}^{(k)}\right)_{i=0}^{\infty}=\left(\sum_{j=k}^{\infty} \frac{1}{r}\left(-\frac{s}{r}\right)^{j-k} \frac{f_{j+1}^{2}}{f_{k} \cdot f_{k+1}} a_{i j}\right)_{i=0}^{\infty} \in c,
$$

for every fixed $k \in \mathrm{N}$, which shows the neccesity (4.7).

Conversely, suppose that the provisos (4.2), (4.3) and (4.7) ensure and take $x=\left(x_{k}\right)$ in the space $l(F(r, s), p)$. Then, $A x$ exists. We follow up for all $m, n \in \mathrm{N}$ that

$$
\begin{aligned}
& \sum_{k=0}^{m}\left|\sum_{j=k}^{n} \frac{1}{r}\left(-\frac{s}{r}\right)^{j-k} \frac{f_{j+1}^{2}}{f_{k} \cdot f_{k+1}} a_{n j} B^{-1}\right|^{q_{k}} \\
& \leq \sup _{n \in \mathrm{N}} \sum_{k}\left|\sum_{j=k}^{\infty} \frac{1}{r}\left(-\frac{s}{r}\right)^{j-k} \frac{f_{j+1}^{2}}{f_{k} \cdot f_{k+1}} a_{n j} B^{-1}\right|^{q_{k}}<\infty
\end{aligned}
$$

which gives the fact that by letting $m, n \rightarrow \infty$ with (4.3) and (4.7)

$$
\begin{aligned}
& \lim _{m, n \rightarrow \infty} \sum_{k=0}^{m}\left|\sum_{j=k}^{n} \frac{1}{r}\left(-\frac{s}{r}\right)^{j-k} \frac{f_{j+1}^{2}}{f_{k} \cdot f_{k+1}} a_{n j} B^{-1}\right|^{q_{k}} \\
& \leq \sup _{n \in \mathrm{N}} \sum_{k}\left|\sum_{j=k}^{\infty} \frac{1}{r}\left(-\frac{s}{r}\right)^{j-k} \frac{f_{j+1}^{2}}{f_{k} \cdot f_{k+1}} a_{n j} B^{-1}\right|^{q_{k}}<\infty .
\end{aligned}
$$

This shows that $\sum_{k}\left|\alpha_{k} B^{-1}\right|^{q_{k}}<\infty$ and $\left(\alpha_{k}\right) \in\{l(F(r, s), p)\}^{\beta}$ which implies that the series $\sum_{k} \alpha_{k} x_{k}$ converges for all $x \in\{l(F(r, s), p)\}$. 
Now, let us consider the equality acquired from 4.5 with $\left(a_{n j}-\alpha_{j}\right)$ instead of $a_{n j}$

$$
\sum_{j}\left(a_{n j}-\alpha_{j}\right) x_{j}=\sum_{k} \sum_{j=k}^{\infty} \frac{1}{r}\left(-\frac{s}{r}\right)^{j-k} \frac{f_{j+1}^{2}}{f_{k} \cdot f_{k+1}}\left(a_{n j}-\alpha_{j}\right) y_{k}=\sum_{k} c_{n k} y_{k}
$$

where $c=\left(c_{n k}\right)$ described by $c_{n k}=\sum_{j=k}^{\infty} \frac{1}{r}\left(-\frac{s}{r}\right)^{j-k} \frac{f_{j+1}^{2}}{f_{k} \cdot f_{k+1}}\left(a_{n j}-\alpha_{j}\right)$ for all $k, n \in$ N. From Lemma 3.3, $c_{n k} \rightarrow 0$, as $n \rightarrow \infty$. This means that $A x \in c$, whenever $x \in l(F(r, s), p)$ and this step completes the proof.

Corollary 4.1. The following expressions are ensured:

i) Let $0<p_{k} \leq 1$ for all $k \in N$. Then, an infinite matrix $A$ transforms all sequences belong to $l(F(r, s), p)$ into $c_{0}$ iff (4.1), and (4.2) ensure and (4.7) ensures with $\alpha_{k}=0$, for all $k \in N$.

ii) Let $1<p_{k} \leq H<\infty$, for all $k \in N$. Then, an infinite matrix $A$ transforms all sequences belong to $l(F(r, s), p)$ into $c_{0}$ iff (4.1), and (4.2) ensure and (4.7) also ensures with $\alpha_{k}=0$, for all $k \in N$.

Lemma 4.1. [1] Let $\lambda, \mu$ be any two sequence spaces, $A$ be an infinite matrix and $B$ be a triangle matrix. Then, $A \in\left(\lambda: \mu_{B}\right)$ iff $B A \in(\lambda: \mu)$.

By considering Theorem, Theorem and Lemma together, it can be acquired following outcome:

Corollary 4.2. Let $A=\left(a_{n k}\right)$ be an infinite matrix of complex constituent. Then, the following expressions are ensured:

i) $E=\left(e_{n k}\right) \in\left(l(F(r, s), p): b v_{\infty}\right)$ iff (4.1), (4.3) ensure with $d_{n k}$ instead of $a_{n k}$; where $d_{n k}=e_{n k}-e_{n-1, k}$ for all $k, n \in N$.

ii) $E=\left(e_{n k}\right) \in\left(l(F(r, s), p): e_{\infty}^{r}\right)$ iff (4.1), (4.3) ensure with $d_{n k}$ instead of $a_{n k} ;$ where $d_{n k}=\sum_{j=0}^{n}\left(\begin{array}{c}n \\ j\end{array}\right)(1-r)^{n-j} r^{j} e_{j k}$ for all $k, n \in N$.

iii) $E=\left(e_{n k}\right) \in\left(l(F(r, s), p): X_{\infty}\right)$ iff (4.1), (4.3) ensure with $d_{n k}$ instead of $a_{n k}$; where $d_{n k}=\sum_{j=0}^{n} \frac{e_{j k}}{(n+1)}$ for all $k, n \in N$.

iv) $E=\left(e_{n k}\right) \in\left(l(F(r, s), p): r_{\infty}^{t}\right)$ iff (4.1), (4.3) ensure with $d_{n k}$ instead of $a_{n k}$; where $d_{n k}=\sum_{j=0}^{n} t_{j} e_{j k} / T_{n}$ for all $k, n \in N$.

v) $E=\left(e_{n k}\right) \in(l(F(r, s), p): b s)$ iff (4.1), (4.3) ensure with $d_{n k}$ instead of $a_{n k} ;$ where $d_{n k}=\sum_{j=0}^{n} e_{j k}$ for all $k, n \in N$.

vi) $E=\left(e_{n k}\right) \in\left(l(F(r, s), p): l_{\infty}(\widehat{F})\right)$ iff (4.1), (4.3) ensure with $d_{n k}$ instead of $a_{n k} ;$ where $d_{n k}=s \frac{f_{n+1}}{f_{n}} e_{n-1, k}+r \frac{f_{n}}{f_{n+1}} e_{n k}$ for all $k, n \in N$.

Corollary 4.3. Let $A=\left(a_{n k}\right)$ be an infinite matrix of complex constituent. Then, the following expressions are ensured: 
i) $E=\left(e_{n k}\right) \in(l(F(r, s), p): c(\Delta))$ iff (4.1), (4.3) and (4.5) with $d_{n k}$ instead of $a_{n k}$; where $d_{n k}=e_{n k}-e_{n+1, k}$ for all $k, n \in N$.

ii) $E=\left(e_{n k}\right) \in\left(l(F(r, s), p): e_{c}^{r}\right)$ iff (4.1), (4.3) and (4.5) also ensures with $d_{n k}$ instead of

where $d_{n k}=\sum_{j=0}^{n}\left(\begin{array}{c}n \\ j\end{array}\right)(1-r)^{n-j} r^{j} e_{j k}$ for all $k, n \in N$.

iii) $E=\left(e_{n k}\right) \in(l(F(r, s), p): \widetilde{c})$ iff (4.1), (4.3) and (4.5) also ensures with $d_{n k}$ instead of $a_{n k}$; where $d_{n k}=\sum_{j=0}^{n} \frac{e_{j k}}{(n+1)}$ for all $k, n \in N$.

iv) $E=\left(e_{n k}\right) \in\left(l(F(r, s), p): r_{c}^{t}\right)$ iff (4.1), (4.3) and (4.5) also ensures with $d_{n k}$ instead of $a_{n k}$; where $d_{n k}=\sum_{j=0}^{n} t_{j} e_{j k} / T_{n}$ for all $k, n \in N$.

v) $E=\left(e_{n k}\right) \in(l(F(r, s), p): c(\widehat{F}))$ iff (4.1), (4.3) and (4.5) also ensures with $d_{n k}$ instead of $a_{n k}$; where $d_{n k}=s \frac{f_{n+1}}{f_{n}} e_{n-1, k}+r \frac{f_{n}}{f_{n+1}} e_{n k}$ for all $k, n \in N$.

vi) $E=\left(e_{n k}\right) \in(l(F(r, s), p): c s)$ iff (4.1), (4.3) and (4.5) also ensures with $d_{n k}$ instead of $a_{n k}$; where $d_{n k}=\sum_{j=0}^{n} e_{j k}$ for all $k, n \in N$.

Corollary 4.4. Let $A=\left(a_{n k}\right)$ be an infinite matrix of complex constituent. Then, the following expressions are ensured:

i) $E=\left(e_{n k}\right) \in\left(l(F(r, s), p): c_{0}(\Delta)\right)$ iff (4.1), (4.3) and (4.5) also ensures with $\alpha_{k}=0$, for all $k \in N$ and $d_{n k}$ instead of $a_{n k}$; where $d_{n k}=e_{n k}-e_{n+1, k}$ for all $k, n \in N$.

ii) $E=\left(e_{n k}\right) \in\left(l(F(r, s), p): e_{0}^{r}\right)$ iff (4.1), (4.3) and (4.7) also ensures with $\alpha_{k}=0$ for all $k \in N$ and $d_{n k}$ instead of $a_{n k}$; where $d_{n k}=\sum_{j=0}^{n}\left(\begin{array}{c}n \\ j\end{array}\right)(1-r)^{n-j} r^{j} e_{j k}$ for all $k, n \in N$.

iii) $E=\left(e_{n k}\right) \in\left(l(F(r, s), p): \widetilde{c_{0}}\right)$ iff (4.1), (4.3) and (4.7) also ensures with $\alpha_{k}=0$ for all $k \in N$ and $d_{n k}$ instead of $a_{n k}$; where $d_{n k}=\sum_{j=0}^{n} \frac{e_{j k}}{(n+1)}$ for all $k, n \in N$.

iv) $E=\left(e_{n k}\right) \in\left(l(F(r, s), p): r_{0}^{t}\right)$ iff (4.1), (4.3) and (4.7) also ensures with $\alpha_{k}=0$ for all $k \in N$ and $d_{n k}$ instead of $a_{n k}$; where $d_{n k}=\sum_{j=0}^{n} t_{j} e_{j k} / T_{n}$ for all $k, n \in N$.

v) $E=\left(e_{n k}\right) \in\left(l(F(r, s), p): c_{0}(\widehat{F})\right)$ iff (4.1), (4.3) and (4.7) also ensures with $\alpha_{k}=0$ for all $k \in N$ and $d_{n k}$ instead of $a_{n k}$; where $d_{n k}=s \frac{f_{n+1}}{f_{n}} e_{n-1, k}+$ $r \frac{f_{n}}{f_{n+1}} e_{n k}$ for all $k, n \in N$.

vi) $E=\left(e_{n k}\right) \in\left(l(F(r, s), p): c_{0} s\right)$ iff (4.1), (4.3) and (4.7) also ensures with $\alpha_{k}=0$ for all $k \in N$ and $d_{n k}$ instead of $a_{n k}$; where $d_{n k}=\sum_{j=0}^{n} e_{j k}$ for all $k, n \in N$.

\section{REFEREN CES}

1. F. BAŞAR and B. Altay: On the spaces of sequences of p-bounded variation and related matrix mappings. Ukrainian Math. J. 55:1 (2003),136-147. 
2. P. N. NG. and P. Y. LeE: TCesaro sequence spaces of non-absolute type. Comment. Math. Prace Mat. 20:2 (1978), 429-433.

3. M. CAndan and E. E. KarA: A Study On topological and geometrical characteristics of new Banach Sequence Spaces. Gulf Journal Of Mathematics 3:4 (2015), 67-84.

4. E. E. KarA: Some topological and geometrical properties of new Banach sequence spaces.J. Inequal. Appl. 38 (2013), 1-15.

5. H. Kizmaz: On certain sequence spaces. Canad. Math. Bull. 24:2 (1981), 169-176.

6. B. Altay, F. Başar and M. Mursaleen : On the Euler sequence spaces which include the spaces $l_{p}$ and $l_{\infty}$. Inform. Sci. 176:10 (2006), 1450-1462.

7. B. Altay and F. BAŞAR : On the Paranormed Riesz sequence spaces of non-absolute type. Southeast Asian Bull. Math.26:5 (2002), 701-715.

8. B. Altay and F. BAŞAR : Some Euler sequence spaces of non-absolute type. Ukrainian Math. J. 57:1 (2005), 1-17

9. M. ŞENGÖNÜL and F. BAŞAR : Some new Cesaro sequence spaces of non-absolute type which include the spaces $c_{0}$ and $c$. Soochow J. Math. 31:1 (2005), 107-119.

10. B. Altay and F. BAŞAR : Some Paranormed Riesz sequence spaces of non-absolute type. Southeast Asian Bull. Math. 30:5 (2006), 591-608.

11. M. BAŞARIR, F. BAŞAR and E. E. KARA: On the spaces of Fibonacci Difference null and convergent sequences. arXiv:1309.0150v1 [math. FA], 2013.

12. B. Choudhary and S. NAnda: Functional Analysis with Applications. JohnWiley, Sons, NewDelhi, India, 1989.

13. I. J. MAdDOX: Spaces of strongly summable sequences.Quart. J. Math. Oxford. 18:2 (1967), 345-355.

14. S. Simons: The sequence spaces $\ell\left(p_{v}\right)$ and $m\left(p_{v}\right)$. Proc. London Math. Soc. 15:3 (1965), 422-436.

15. H. NAKAno: Modulared sequence spaces. Proc. Japan Acad. 27:2 (1951), 508-512.

16. M. Mursaleen: On some geometric properties of a sequence space related to $l_{p}$. Bull. Aust. Math. Soc. 67 (2003), 343-347.

17. C. Aydin and F. BAŞAR: Some new sequence spaces which include the spaces $l_{p}$ and $l_{\infty}$. Demonstr. Math. 38 (2005), 641-656.

18. B. ALtAY and F. BAŞAR: The matrix domain and the fine spectrum of the difference operator $\Delta$ on the sequence space $l_{p} ;(0<p<1)$. Commun. Math. Anal. 2 (2007), $1-11$.

19. F. BAŞAR: Summability Theory and Its Applications,. Bentham Science Publishers, e-books, Monographs, xi+405 pp., İstanbul, (2012) ISB:978-1-60805-252-3,

20. M. Mursaleen and A. K. Noman : On some new sequence spaces of non-absolute type related to the spaces $l_{p}$ and $l_{\infty}, I$. Filomat. 25:2 (2011), 33-51.

21. M. Mursaleen and A. K. Noman: On some new sequence spaces of non-absolute type related to the spaces $l_{p}$ and $l_{\infty}, I I$. Mathematical Communications. 16:2 (2011), 383-398.

22. M. Candan and K. Kayaduman: Almost convergent sequence space derived by generalized Fibonacci matrix and Fibonacci core. Brithish J. Math. Comput. Sci7:2 (2015), 150-167. 
23. M. CANDAN: A new sequence space isomorphic to the space $l(p)$ and compact operators. J. Math. Comput. Sci 4:2 (2014), 306-334.

24. M. CANDAN: A new approach on the spaces of generalized Fibonacci difference null and convergent sequences. Math. Aeterna. 1:5 (2015), 191-210.

25. A. Alotaibi, M. Mursaleen, B. A. Alamri and S. A. Mohiuddine: Compact operators on some Fibonacci difference sequence spaces. Journal of Inequalities and Applications. (2015), 2015:203 DOI10.1186s13660-015-0713-5.

26. M. Candan: Domain of Double Sequential Band Matrix in the Spaces of Convergent and Null Sequences. Advanced in Difference Equations. 1 (2014), 1-18.

27. E. Malkowsky and V. RakočEvić: An Introduction into the theory of sequence spaces and measures of noncompactness. Zbornik Radova, Matematički Institut SANU, Belgrade, 9:17 (2000), 143-234.

28. A. Wilansky: Functional Analysis. Blaisdell Publishing Company, New YorkToronto-London, 1964.

29. C. G. LASCARIDES and I. J. MADDOX: Matrix transformations between some classes of sequences. Proc. Camb. Phil. Soc. 68 (1970), 99-104.

30. K. G. Grosse- Erdman: Matrix transformations between the sequence spaces of Maddox. J. Math. Anal. Apply 180 (1993), 223-238.

31. A. JARrah and E. Malkowsky: BK-spaces, bases and linear operators. Rendiconti Circ. Mat. Palermo II 52 (1990), 177-191.

32. H. ÇAPAN and F. BAŞAR: Domain of The Double Band Matrix Defined By Fibonacci Numbers in The Maddox's Space $l(p)$ Electronic Journal of Mathematical Analysis and Applications 3:2 (2015), 31-45.

33. M. CANDAN: Almost convergence and double sequential band matrix. Acta Math. Scientia. 34:2 (2014), 354-366.

34. M. CAndan and A. GünEŞ: Paranormed sequence spaces of Non Absolute Type Founded Using Generalized Difference Matrix. Proceedings of the National Academy of Sciences; India Section A:Physical Sciences. 85:2 (2014), 269-276.

\author{
Gülsen Kılınç \\ Faculty of Education \\ Department of Elementary Education \\ Adıyaman University, \\ The University Campus, \\ 02040-Adıyaman/TURKEY \\ gkilinc@adiyaman.edu.tr
}

